PROCEEDINGS OF THE

AMERICAN MATHEMATICAL SOCIETY

Volume 134, Number 2, Pages 487-490

S 0002-9939(05)08008-1

Article electronically published on July 8, 2005

\title{
MAXIMALITY THEOREMS FOR FRÉCHET ALGEBRAS
}

\author{
ŽELJKO ČUČKOVIĆ AND N. V. RAO
}

(Communicated by Juha M. Heinonen)

\begin{abstract}
The algebra of unbounded holomorphic functions $\bigcap_{p \geq 1} H^{p}(\partial \mathbb{D})$ that is contained in the algebra $\bigcap_{p>1} L^{p}(\partial \mathbb{D})$ is studied. For $f$ in $\bigcap_{p>1} L^{p}(\partial \mathbb{D})$ but not in $\bigcap_{p>1} H^{p}(\partial \mathbb{D})$, we show that the algebra generated by $\bigcap_{p>1} H^{p}(\partial \mathbb{D})$ and $f$ is dense in $L^{p}(\partial \mathbb{D})$ for all $p \geq 1$.
\end{abstract}

\section{INTRODUCTION}

In what follows, let $1 \leq p<\infty$ and let $\mathbb{D}$ be the unit disk in the complex plane C. We are interested in the Fréchet algebra $H=\bigcap_{p \geq 1} H^{p}(\partial \mathbb{D})$, where $H^{p}(\partial \mathbb{D})$ denotes the usual Hardy class of all functions $f \in L^{p}(\partial \bar{D})$ for which

$$
\int_{0}^{2 \pi} f\left(e^{i \theta}\right) e^{i n \theta} d \theta=0 \text { for all } n>0 .
$$

For any $f$ in the Fréchet algebra $L$ defined by $L=\bigcap_{p \geq 1} L^{p}(\partial \mathbb{D})$ but not in $H$, we form the algebra $\mathcal{A}$ generated by $H$ and $f$ in $L$. Our main result is that $\mathcal{A}$ is dense in $L^{p}(\partial \mathbb{D})$ for every $p \geq 1$. Hence the algebra $H$ is maximal in $L$. We use the F. and M. Riesz Theorem along with Beurling's inner and outer function factorization of functions in Hardy classes. In fact our method gives another proof of Wermer's Maximality Theorem [W] without using the Banach algebra techniques: The algebra of functions continuous on $\overline{\mathbb{D}}$ and holomorphic in $\mathbb{D}$ is maximal in the algebra of continuous functions on $\partial \mathbb{D}$. We are sure that these results are true for finitely connected domains and many infinitely connected domains, and this proof can be easily extended. We intend to return to this topic in an expository paper later on.

Maximality theorems were fashionable a long time ago, and we would like to explain how we were led to this theorem at this particular time. In fact the path that led us to this is even more interesting. Hoffman $[\mathrm{H}$ showed that for $f$ in $L^{\infty}(\partial \mathbb{D}) \backslash H^{\infty}(\partial \mathbb{D})$, the norm closed subalgebra of $L^{\infty}(\partial \mathbb{D})$ generated by $H^{\infty}(\partial \mathbb{D})$ and $f$ (usually denoted by $H^{\infty}(\partial \mathbb{D})[f]$ ) contains the algebra of continuous functions $C(\partial \mathbb{D})$. However Axler and Shields $[\mathrm{AS}$ ] proved that for $f$ a bounded harmonic function in $\mathbb{D}$ that is not holomorphic, $H^{\infty}(\mathbb{D})[f]$ contains $C(\overline{\mathbb{D}})$. This theorem was later generalized by Bishop $[\mathrm{B}$ for more general domains.

These theorems of Axler-Shields and Bishop played an important role in a problem involving Toeplitz operators on the Bergman space $L_{a}^{2}(\Omega)$. Here $\Omega$ denotes a

Received by the editors September 16, 2003 and, in revised form, September 23, 2004.

2000 Mathematics Subject Classification. Primary 30H05, 30D55; Secondary 30E10, 46E25.

(C)2005 American Mathematical Society 
bounded domain in $\mathbb{C}$ and let $d A$ denote the Lebesgue area measure on $\Omega$. The Bergman space $L_{a}^{p}(\Omega)$ is the space of all functions $f$ holomorphic on $\Omega$ that satisfy $\int_{\Omega}|f|^{p} d A$ is finite. The space $L_{a}^{2}(\Omega)$ is a closed subspace of $L^{2}(\Omega, d A)$. So there exists the orthogonal projection $P$ from $L^{2}(\Omega)$ onto $L_{a}^{2}(\Omega)$. For a bounded measurable function $\phi$ on $\Omega$, the Toeplitz operator $T_{\phi}$ is the operator on $L_{a}^{2}(\Omega)$ defined by $T_{\phi}(f)=P(\phi f)$.

In AČR it was shown that if $\phi$ is a nonconstant bounded holomorphic function on $\Omega$ and $\psi$ is a bounded measurable function on $\Omega$ such that $T_{\phi} T_{\psi}=T_{\psi} T_{\phi}$, then $\psi$ must be holomorphic too. An important ingredient in the proof was the theorem of Bishop B (or Axler and Shields AS for the open unit disk only).

It is well known that Toeplitz operators on $L_{a}^{2}(\Omega)$ could be bounded even if their symbols are unbounded. Pat Ahern asked if the Axler-Cučković-Rao theorem holds in this situation. For that reason he asked for the following replacement of the Axler-Shields theorem in their proof:

If $A=\bigcap_{p \geq 1} L_{a}^{p}(\Omega)$ and $f \in A$ is not constant, is the algebra generated by $A$ and $\bar{f}$ dense in $L^{p}(\Omega)$ for any $p>1$ ? We could not answer this question, but by replacing $L_{a}^{p}(\Omega)$ by $H^{p}(\partial \mathbb{D})$ and $L^{p}(\Omega)$ by $L^{p}(\partial \mathbb{D})$, we asked the same question and answered positively.

Thus the original question of Ahern is still open. Also the following problems are open:

Let $A=\bigcap_{p \geq 1} L_{a}^{p}(\mathbb{D})$ and $L=\bigcap_{p \geq 1} L^{p}(\mathbb{D})$. Further let $f \in L$ be nowhere holomorphic in $\mathbb{\mathbb { D }}$. Then is the algebra generated by $A$ and $f$ dense in $L$ ?

Can the unit disk $\mathbb{D}$ be replaced by an arbitrary domain $\Omega$ in the above?

\section{Main theorem: Statement And PRoOF}

Theorem 1. Algebra $\mathcal{A}$ is dense in $L^{p}(\partial \mathbb{D})$ for any $p \geq 1$.

Proof. Fix a $p \geq 1$. Let $\alpha$ be any continuous linear functional on $L^{p}(\partial \mathbb{D})$ which vanishes on $\mathcal{A}$. We wish to show that $\alpha=0$. By hypothesis

$$
\alpha\left(z^{k} f^{n}\right)=0 \text { for all } k \geq 0, n \geq 0 .
$$

Further by the Riesz Duality, $\alpha$ is given by a function $g \in L^{q}(\partial \mathbb{D})$ where $\frac{1}{p}+\frac{1}{q}=1$ and

$$
\alpha(h)=\int_{0}^{2 \pi} h\left(e^{i \theta}\right) g\left(e^{i \theta}\right) d \theta
$$

for every $h \in H^{p}$. Now by the F. and M. Riesz theorem and (1), we get that $g f^{n}$ belongs to $H^{1}(\partial \mathbb{D})$ for all $n \geq 0$. If we write $g f^{n}=\mu_{n}$, we have $g=\mu_{0}$ and $g f=\mu_{1}$ so that

$$
\mu_{0} f=\mu_{1} \text { on } \partial \mathbb{D} \text {. }
$$

Using Beurling's inner and outer function factorization of Hardy class functions, we can write

$$
\mu_{n}=B_{n} S_{n} O_{n}
$$

where $B_{n}$ is Blaschke, $S_{n}$ is singular, and $O_{n}$ is outer. So we have

$$
B_{0} S_{0} O_{0} f=B_{1} S_{1} O_{1} \text {. }
$$

From the equation $g f^{n}=\mu_{n}$, we obtain

$$
B_{0} S_{0} O_{0} B_{1}^{n} S_{1}^{n} O_{1}^{n}=B_{0}^{n} S_{0}^{n} O_{0}^{n} B_{n} S_{n} O_{n} \text { on } \partial \mathbb{D} \text { for all } n \geq 0 .
$$


By taking logarithms of the absolute value on both sides of (2), we get

$$
O_{0} O_{1}^{n}=O_{0}^{n} O_{n} \text {. }
$$

Now cancelling the outer functions in (2), by the uniqueness of factorization of Hardy class functions, we conclude that

$$
B_{0} B_{1}^{n}=B_{0}^{n} B_{n}, S_{0} S_{1}^{n}=S_{0}^{n} S_{n} .
$$

Now we claim that $B_{0}$ divides $B_{1}$. For any point $z$ in the unit disk and any function $h$ holomorphic in the unit disk, let $O(z, h)$ denote the order of vanishing of $h$ at $z$. So from (3), we get $O\left(z, B_{0}\right)+n O\left(z, B_{1}\right)=n O\left(z, B_{0}\right)+O\left(z, B_{n}\right)$ for all $z$ and $n \geq 0$, from which it follows that

$$
O\left(z, B_{0}\right)+n O\left(z, B_{1}\right) \geq n O\left(z, B_{0}\right) .
$$

Dividing by $n$ and letting $n$ go to $\infty$, we have $O\left(z, B_{1}\right) \geq O\left(z, B_{0}\right)$ for all $z$ and hence $B_{0}$ divides $B_{1}$.

Let $\nu_{n}$ denote the associated singular measure of the singular function $S_{n}$. Again from (3), we obtain

$$
\nu_{0}+n \nu_{1}=n \nu_{0}+\nu_{n} \text { for all } n \geq 0
$$

which means

$$
\nu_{0}+n \nu_{1} \geq n \nu_{0} \text { for all } n \text {. }
$$

Now again, dividing (4) by $n$ and letting $n \rightarrow \infty$, we get

$$
\nu_{1} \geq \nu_{0} \text {. }
$$

Thus $S_{0}$ divides $S_{1}$ and hence $\frac{B_{1}}{B_{0}}$ is Blaschke, $\frac{S_{1}}{S_{0}}$ is singular. Further since $\frac{O_{1}}{O_{0}}$ is outer, we have that

$$
f=\frac{B_{1}}{B_{0}} \frac{S_{1}}{S_{0}} \frac{O_{1}}{O_{0}}
$$

is the boundary value of a holomorphic function in the Nevanlinna class and since $f \in L^{p}(\partial \mathbb{D})$, it also belongs to the Hardy class $H^{p}(\partial \mathbb{D})$ (see $[\mathbb{R}$, p. 371, Th.17.16), which is a contradiction to the assumption

$$
f \in L \backslash H .
$$

Therefore $g \equiv 0$ and $\mathcal{A}$ is dense in all $L^{p}(\partial \mathbb{D})$ and so dense in $L$ as a Fréchet subalgebra.

Wermer's Maximality Theorem. The algebra of functions $f$ continuous on $\overline{\mathbb{D}}$ and holomorphic in $\mathbb{D}$ is maximal in the algebra $C(\partial \mathbb{D})$ of complex-valued continuous functions on $\partial \mathbb{D}$.

Proof. The proof is exactly the same.

\section{REFERENCES}

[AS] S. Axler and A. Shields, Algebras generated by analytic and harmonic functions, Indiana Univ. Math. J. 36 (1987), 631-638. MR0905614 (88h:46102)

[AČR] S. Axler, Ž. Čučković, and N. V. Rao, Commutants of analytic Toeplitz operators on the Bergman space, Proc. Amer. Math. Soc. 128 (2000) no.7, 1951-1953. MR1694299 (2000m:47035)

[B] C. Bishop, Approximating continuous functions by holomorphic and harmonic functions, Trans. Amer. Math. Soc. 311 (1989), 781-811. MR0961619 (89j:30051)

[H] K. Hoffman, Banach Spaces of Analytic Functions, Dover, New York, 1988. MR1102893 (92d:46066) 
[R] W. Rudin, Real and Complex Analysis 2nd ed., McGraw-Hill, 1974. MR0344043 (49:8783)

[W] J. Wermer, On algebras of continuous functions, Proc. Amer. Math. Soc. 4 (1953), 866869. MR0058877 (15:440g)

Department of Mathematics, The University of Toledo, Toledo, Ohio 43606

E-mail address: zcuckovi@math.utoledo.edu

Department of Mathematics, The University of Toledo, Toledo, Ohio 43606

E-mail address: rnagise@math.utoledo.edu 